EPJ Web of Conferences 57, 02002 (2013)

DOI: $10.1051 /$ epjconf/20135702002

(C) Owned by the authors, published by EDP Sciences, 2013

\title{
A gravitational wave detector operating beyond the quantum shot-noise limit: Squeezed light in application
}

\author{
Roman Schnabela (for the LIGO Scientific Collaboration) \\ Institut für Gravitationsphysik, Leibniz Universität Hannover, and Max-Planck-Institut für \\ Gravitationsphysik, Callinstr. 38, 30167 Hannover, Germany
}

\begin{abstract}
This contribution reviews our recent progress on the generation of squeezed light [1], and also the recent squeezed-light enhancement of the gravitational wave detector GEO 600 [2]. GEO 600 is currently the only GW observatory operated by the LIGO Scientific Collaboration in its search for gravitational waves. With the help of squeezed states of light it now operates with its best ever sensitivity, which not only proves the qualification of squeezed light as a key technology for future gravitational wave astronomy but also the usefulness of quantum entanglement.
\end{abstract}

\section{INTRODUCTION}

Einstein's general theory of relativity predicts that non-axisymmetric acceleration of matter creates oscillations of space-time geometry that propagate at the speed of light. In the far field these oscillations are called gravitational waves (GWs) and are, to lowest order, a transversal quadrupole radiation. Prominent sources of GWs are coalescing neutron star and black hole binaries, supernovae, and the big bang [3]. The detection of GWs with a high signal-to-noise ratio will open a completely new window to our universe through which even electro-magnetically dark objects can be studied. The detection of GWs is, however, extremely challenging and has not been achieved so far because expected amplitudes are tiny for large distances.

Current gravitational wave detectors are Michelson-type kilometer-scale laser interferometers and measure the distance changes between quasi-free mirrors. The sensitivity of these detectors at frequencies above several hundred hertz is limited by the vacuum (zero-point) fluctuations of the light mode used. As pointed out by C.M. Caves [4], the vacuum fluctuations that enter the Michelson interferometer at its signal output port that is close to a dark fringe disturb the GW detection in two ways. First, it leads to an uncertainty of the differential amplitude quadrature of the light fields inside the interferometer arms $\left(\hat{X}_{1}\right)$. This noise produces a stochastic radiation pressure force that drives the differential mirror motion. Second, the vacuum fluctuations in the orthogonal quadrature lead to an uncertainty of the differential phase quadrature of the light fields inside the interferometer arms $\left(\hat{X}_{2}\right)$, which corresponds to the photon counting statistics (shot noise) at the interferometer's output port. The two types of opto-mechanical and purely optical quantum noise, respectively, can be expressed as spectral densities normalized to the differential mirror displacement or normalized to the strain produced by a gravitational wave. For a Michelson interferometer without optical cavities the relative shot-noise spectral density has a white spectrum, while the relative radiation pressure noise spectral density falls with $1 / f^{2}$ above the pendulum resonance (of the order of $1 \mathrm{~Hz}$ ), see dashed lines in Fig. 1. The shot-noise spectral density decreases with increasing light power $(\propto 1 / \sqrt{2})$ and corresponds to

\footnotetext{
ae-mail: roman.schnabel@aei.mpg.de
}

This is an Open Access article distributed under the terms of the Creative Commons Attribution License 2.0, which permits unrestricted use, distribution, and reproduction in any medium, provided the original work is properly cited. 


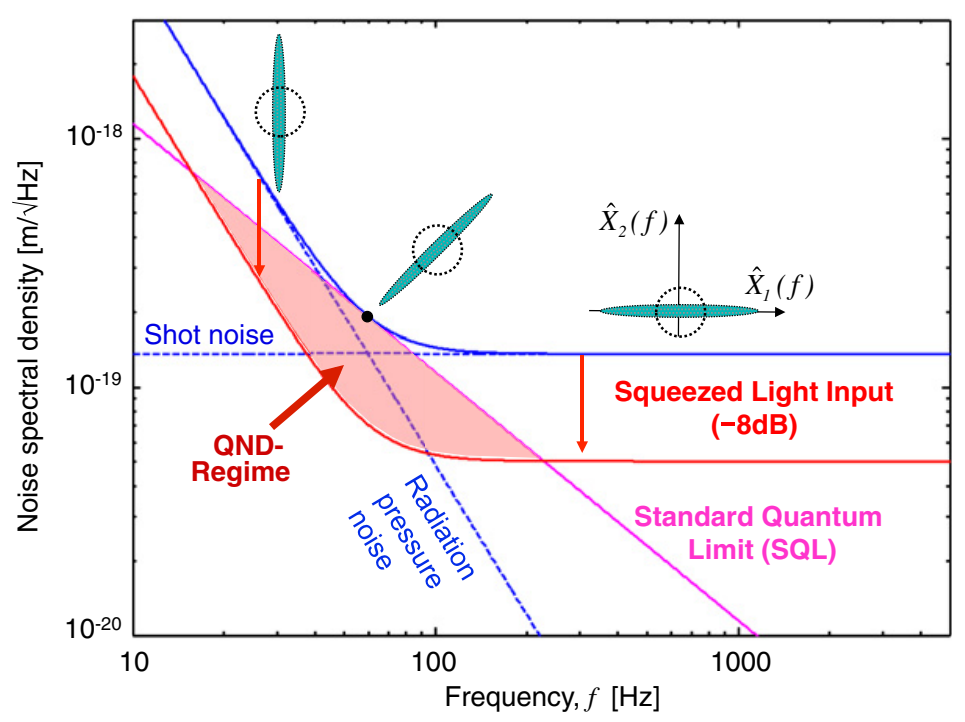

Figure 1. Examplary quantum noise spectral density for a simple Michelson laser interferometer with quasi-free, pendulum suspended mirrors using a simple single photo diode readout (solid blue line). The interferometer's standard-quantum-limit (SQL, straight solid line) takes into account the mirror masses and defines the frequencydependent lower bound of the noise spectral density that can be achieved by varying the light power. The SQL can be surpassed by injecting a broadband, frequency-dependent squeezed state of light [8] into the interferometer's signal port thereby turning the interferometer into a quantum non-demolition device [11]. The red solid line assumes an injected squeezed vacuum with a variance being a factor of $6.3(8 \mathrm{~dB})$ below that of the vacuum noise. Broadband squeezed light with a frequency-dependent squeezing angle was demonstrated in [12].

quantum measurement noise, while the radiation pressure noise spectral density increases with light power $(\propto \sqrt{2})$ and corresponds to quantum back-action noise. The two quantum noise contributions can both be reduced by replacing the ordinary vacuum field entering the interferometer's signal port by a broadband squeezed vacuum state [5]. Most importantly, an appropriately designed broadband squeezed state is even able to simultaneously squeeze the two noise contributions [6-8]. The three ellipses in Fig. 1 represent Wigner functions of the optimized broadband squeezed field that simultaneously reduces the dominating quantum noise at radiation pressure noise dominated frequencies (left), at the SQL (center), and at shot-noise dominated frequencies (right). Recent reviews on the application of quantum optics and optomechanics in GW detectors are given in Refs. [9, 10].

\section{IMPROVING THE SIGNAL-TO-SHOT-NOISE RATIO}

Today's GW detectors are limited by shot noise at frequencies above a few hundreds of hertz, whereas back action noise has not yet been observed due to technical noise sources at low frequencies. In the past decades several proposals were made and realized in GW detectors to reduce the shot noise. Apart from higher input light powers, all first generation GW detectors use enhancement ("recycling") cavities [15] as shown in Fig. 2. At frequencies above a few hundreds of hertz, however, all detectors are still limited by shot noise. Increasing the light power is becoming more and more difficult. Ever higher light powers will increase the risk of damaging laser optical components. Further increasing the thermal load at the interferometer's beam splitter will result in a significant thermal lense that drives the interferometer out of its optical stability regime. Further increasing the finesse values of the arm cavities will narrow the detection band of the detectors. The second generation of GW detectors, which is currently under construction, will eventually be partially limited by radiation pressure noise. In this 

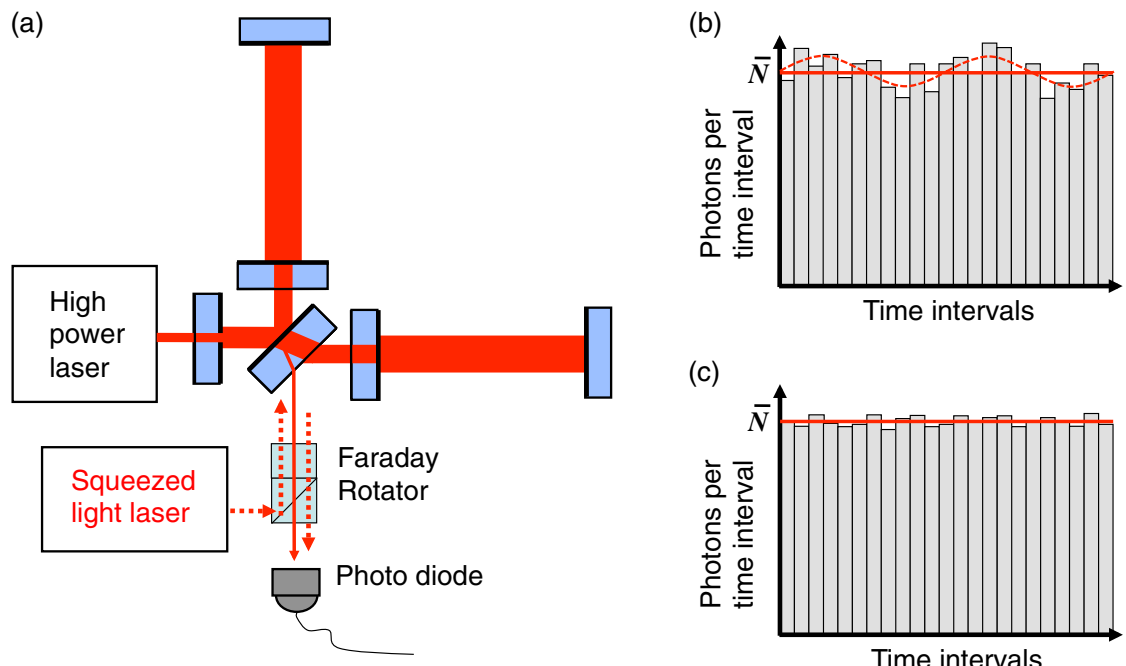

Time intervals

Figure 2. (a) Interferometric GW detectors use high power light fields to reduce the relative shot noise. Limitations occur because the arm cavity linewidth needs to be larger than the desired signal band, and an excessive thermal load on the beam splitter and the arm cavity couplers produce thermal lenses that eventually drive the cavities into optical instability. Squeezed vacuum states of light injected into the signal port (being close to a dark fringe) of the interferometer allow a reduction of the relative shot-noise without increasing the light power inside the interferometer [5]. The squeezed states split at the interferometer's beam splitter and result in entanglement between the high power light fields in the arms as previously verified in table-top experiments [13, 14]. If a frequencydependent phase space rotation is applied to the squeezed field by filter cavities [11] (not shown) radiation pressure noise can be simultaneously squeezed as shown in Fig. 1. (b) Representation of the photon counting statistic of a coherent light field detected by a photo diode. The dashed line corresponds to an apparent signal due to shot noise. (c) Reduced standard deviation due to the interference between a coherent light field and a broadband squeezed vacuum field.

case a further increase of light power fundamentally is of limited use. Also low-temperature operation of future GW detectors, aiming for an reduction of thermally excited motion of mirror surfaces, demands the utilization of squeezed states of light, in combination with a tolerable high power (coherent) light field.

The right side of Fig. 2 shows the effect of a broadband squeezed vacuum field injected into the interferometer's signal port on the photon counting statistics at the photo diode. Inside the interferometer the injected squeezed states produce quantum correlations between the phase quadratures of the light fields in the arms, and thus entanglement as previously demonstrated in table-top experiments $[13,14]$.

\section{GENERATION OF SQUEEZED LIGHT FOR GRAVITATIONAL WAVE DETECTION}

Squeezed light was first produced in the mid 1980s [16-18]. In these first experiments, squeezing below the shot-noise variance of a few percent up to a factor of $2(3 \mathrm{~dB})$ was observed, at frequencies above the audio-band. GW detectors require strong broadband squeezing over the full GW detection band from $10 \mathrm{~Hz}$ to $10 \mathrm{kHz}$. In 2003 I developed the idea to build a squeezed-light source for $\mathrm{GW}$ detection. A review article on the progress in the field is given by Ref. [9]. Important milestones were the controlled generation of squeezed states at audio-band frequencies [19-21], the test of compatibility of squeezedlight injection with enhancement cavities [22-26], and the generation of strongly squeezed states of light [27-32].

Figure 3 shows a photograph of our squeezed-light laser that was completed in 2010 at the Institute for Gravitational Physics (Albert-Einstein-Institute Hannover) and that is now part of the GEO 600 


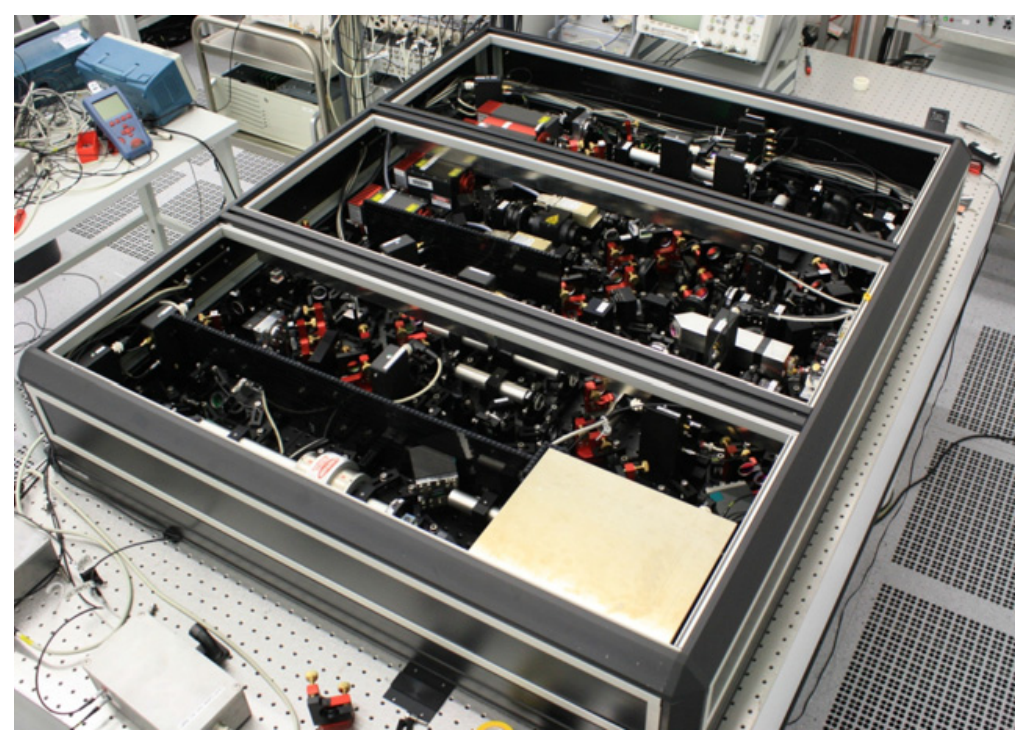

Figure 3. Photograph of the GEO 600 squeezed-light laser $(135 \mathrm{~cm} \times 113 \mathrm{~cm})$ [1]. Three Nd : YAG lasers (upper left part) provide continuous-wave light at $1064 \mathrm{~nm}$ that is used to generate second harmonic pump light for the parametric generation of broadband squeezed vacuum states of light $[18,28]$ and the control beams for stabilizing the squeezing angle with respect to the GEO 600 main laser. The arrow points toward the aluminum housing of the squeezing resonator having dimensions of about $3 \mathrm{~cm} \times 6 \mathrm{~cm} \times 6 \mathrm{~cm}$.

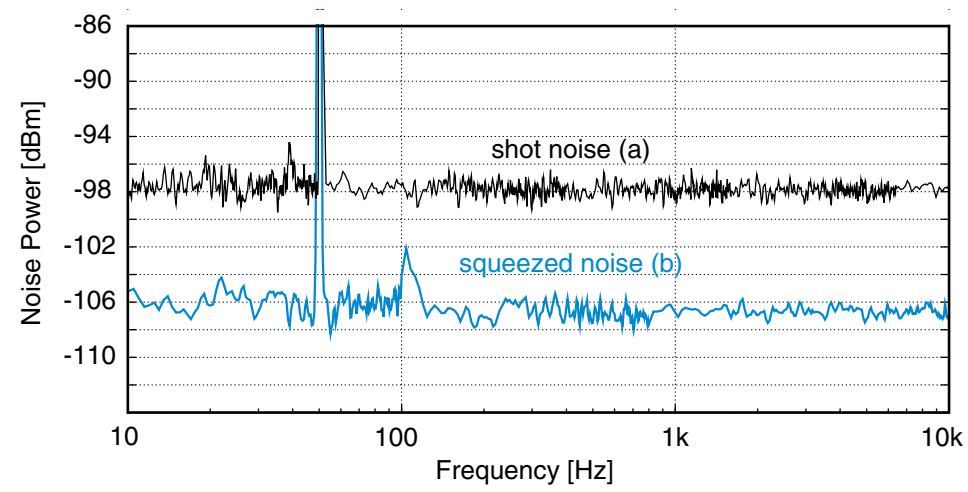

Figure 4. Quantum noise measurements on the squeezed-light laser performed with a balanced homodyne detector (BHD) [1]. Trace (a) corresponds to the shot-noise (vacuum noise) reference of our BHD, measured with the squeezed-light input blocked. Trace (b) shows the observed squeezed quantum noise. A nonclassical noise suppression of up to $9 \mathrm{~dB}$ below shot-noise was measured throughout the spectrum from $10 \mathrm{~Hz}$ to $10 \mathrm{kHz}$. The electronic dark noise (not shown) was $17 \mathrm{~dB}$ below the shot-noise and was not subtracted from the data shown. The peaks at $50 \mathrm{~Hz}$ and $100 \mathrm{~Hz}$ were due to the electric mains supply.

detector [33-35]. It is realized on a $1.5 \mathrm{~m}^{2}$ breadboard and fully automated on the basis of a real-time control system. Figure 4 shows the measured quantum noise reduction of this laser, taken over the full detection band of ground-based GW detectors. More details can be found in [1, 36-38]. 


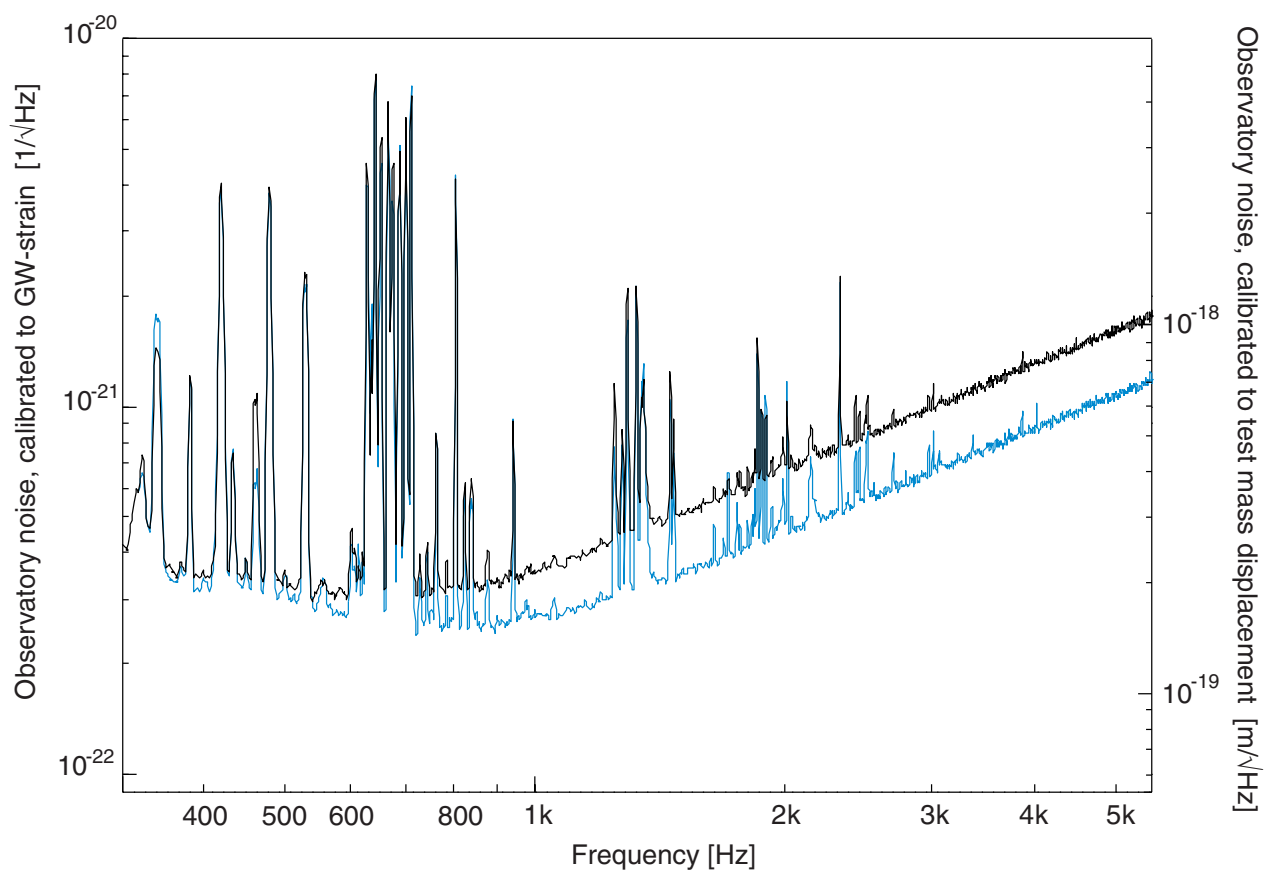

Figure 5. Nonclassical reduction of the GEO 600 instrumental noise as reported in [2]. The noise is calibrated to GW-strain as well as differential mirror displacement, respectively. The upper curve shows the observatory noise spectral density without the injection of squeezed light; at frequencies above $700 \mathrm{~Hz}$ GEO 600 is shot-noise limited. Note that the slope in the kHz-regime is due to the frequency-dependent signal enhancement of the signal-recycling cavity. The injection of squeezed vacuum states leads to a broadband noise reduction of up to $3.5 \mathrm{~dB}$ (lower curve). The spectral features are caused by excited violin modes of the suspensions $(600-700 \mathrm{~Hz}$ and harmonics) as well as by calibration $(160 \mathrm{~Hz}-2.5 \mathrm{kHz})$. The broad unresolved noise structures from about $120 \mathrm{~Hz}$ to $220 \mathrm{~Hz}$ are caused by insufficient seismic isolation of mirrors located between the signal recycling mirror and the output mode-cleaner. (Measurement data: courtesy by H. Grote and the LIGO Scientific Collaboration (LSC)).

\section{SQUEEZED LIGHT ENHANCEMENT OF THE GW DETECTOR GEO 600}

The German-British GEO 600 detector is one of the large-scale Michelson interferometers searching for gravitational wave signals and is located about $20 \mathrm{~km}$ south of Hannover [33-35]. GEO 600 does not have arm cavities but a signal-recycling (SR) mirror placed in reflection of the almost dark signal output port. GEO 600 is currently operated with a carrier-tuned signal-recycling cavity [15] to symmetrically enhance the upper and lower signal sidebands. The output field is detected by a single photo diode as shown in Fig. 2. The output light transmitted by the SR mirror is filtered with an output mode-cleaner cavity (OMC) [39], which suppresses technical modulation sidebands at radio-frequencies, as well as spurious light modes originating from mirror imperfections. Both filter effects assure that the detected beam is not contaminated by additional technical or quantum noise.

In April 2010, the squeezed-light laser was brought to the GEO 600 site. In the subsequent months it was integrated into the complex optical arrangement of GEO 600 under the guidance of H. Grote and H. Vahlbruch. The GEO 600 team were facing several tasks. In particular, a stable phase lock loop between the main high-power laser and the squeezed-light laser had to be realized, and the squeezed mode matched into the signal-recycling cavity of GEO 600 using a low-loss custom-made Faraday rotator. Also the new interferometer input port used for squeezed-light injection had to be protected against back-reflection of scattered and frequency shifted photons [21]. Figure 5 presents the improvement of the GEO 600 noise spectral density when the squeezed-light injection was completed 


\section{EPJ Web of Conferences}

after a couple of months of commissioning. The figure shows a broadband noise reduction of up to $3.5 \mathrm{~dB}$ (black to blue trace) in the shot-noise limited frequency band (above $700 \mathrm{~Hz}$ ). At $3 \mathrm{kHz}$, the quantum noise was reduced from $1.0 \times 10^{-21} \mathrm{~Hz}^{-1 / 2}$ down to $6.7 \times 10^{-22} \mathrm{~Hz}^{-1 / 2}$. By now, the squeezing enhancement has been successfully operated over several months. Due to squeezed light GEO 600 now achieves its best ever sensitivity. At frequencies below $700 \mathrm{~Hz}$ GEO 600 is limited by technical noise which is not reduced by quantum noise squeezing. Note that quantum radiation pressure noise [4] is currently not dominant in any band of the spectral density. The maximum nonclassical quantum noise reduction in GEO 600 is currently not limited by the squeezed-light laser but by optical loss on the squeezed light during propagation in the interferometer. About $10 \mathrm{~dB}$ of squeezing is injected but the degree of squeezing degrades by photon absorption and scattering inside the GEO 600 signal recycling cavity and the output mode-cleaner, both contributing about $10 \%$ loss. In addition the nonperfect photo diode quantum-efficiency, the absorption of the Faraday isolators and auxiliary optics, and finally some residual mode mismatch cause an additional $20 \%$ loss. The total loss corresponds to an overall optical efficiency of $\eta=0.62$. It leads to an increase of the squeezed noise variance from $V_{\mathrm{sqz}}=0.1$ to $\eta V_{\mathrm{sqz}}+(1-\eta)=0.44$, and to a corresponding attenuation of the squeezing factor from $10 \mathrm{~dB}$ to $3.5 \mathrm{~dB}$, which is in excellent agreement with our results presented in [2] and shown in Figure 5.

\section{CONCLUSION AND OUTLOOK}

The progress in the generation of squeezed laser light now enables a true application of squeezed light, as realized in the gravitational wave detector GEO 600. Since 2010 GEO 600 has been using squeezed light during its searches for gravitational waves [40]. In the meantime also a succesful test of squeezed light in one of the LIGO detectors was performed [41]. The observed nonclassical improvement of up to $3.5 \mathrm{~dB}$ in [2] has been limited by optical loss inside the interferometer. Designs of future GW detectors such as the Einstein Telescope [42, 43] will significantly reduce this loss and a nonclassical improvement of $10 \mathrm{~dB}$ with state-of-the-art squeezed light lasers is expected. My conjecture is that all future GW detectors will be enhanced with squeezed light. Squeezed light solves the problem of increasing the measurement sensitivity without increasing the light power. This issue is already important for GEO 600 since its beam splitter shows thermal lensing due to absorbed light. The thermal load on optical components due to high light powers, however, will become even more important for future GW detectors with cryogenically cooled test mass mirrors such as the Japanese detector KAGRA [44]. Squeezed light allows the reduction of relative quantum noise without increasing the number of quanta. Due to this nonclassical property, squeezed light will contribute to the endeavor of making GW astronomy a reality.

I thank current and previous members of my group, in particular the following who all contributed to improve our squeezed light sources: Stefan Ast, Jöran Bauchrowitz, Christof Baune, Simon Chelkowski, James DiGuglielmo, Tobias Eberle, Alexander Franzen, Christian Gräf, Vitus Händchen, Boris Hage, Aleksandr Khalaidovski, Nico Lastzka, Moritz Mehmet, Aiko Samblowski, Sebastian Steinlechner, André Thüring, Henning Vahlbruch, and Christina Vollmer. I also thank Hartmut Grote and the GEO 600 team for their great work regarding the implementation of the squeezed-light source in GEO 600.

\section{References}

[1] H. Vahlbruch, A. Khalaidovski, N. Lastzka, C. Gräf, K. Danzmann, R. Schnabel, Class. Quantum Grav. 27, 084027 (2010).

[2] The LIGO Scientific Collaboration, Nature Physics 7, 962 (2011). 
[3] B. S. Sathyaprakash and B.F. Schutz, Living Rev. Relativity 12, 2 (2009).

[4] C. M. Caves, Phys. Rev. Lett. 45, 75 (1980).

[5] C. M. Caves, Phys. Rev. D 23, 1693 (1981).

[6] W. G. Unruh, in Quantum Optics, Experimental Gravitation, and Measurement Theory, edited by P. Meystre and M. O. Scully (Plenum, New York, 1983), 647-660.

[7] H. P. Yuen, Phys. Rev. Lett. 51, 719 (1983); Phys. Rev. Lett. 52 (1984).

[8] M. T. Jaekel and S. Reynaud, Europhys. Lett. 13, 301 (1990).

[9] R. Schnabel, N. Mavalvala, D. E. McClelland, P. K. Lam, Nat. Commun. 1, 121 (2010).

[10] D. E. McClelland, N. Mavalvala, Y. Chen, R. Schnabel, Laser Photonics Rev. 5, 677 (2011).

[11] H. J. Kimble, Yu. Levin, A. B. Matsko, K. S. Thorne, and S. P. Vyatchanin, Phys. Rev. D 65, 022002 (2001).

[12] S. Chelkowski, H. Vahlbruch, B. Hage, A. Franzen, N. Lastzka, K. Danzmann, R. Schnabel, Phys. Rev. A 71, 013806 (2005).

[13] J. DiGuglielmo, B. Hage, A. Franzen, J. Fiurášek, and R. Schnabel, Phys. Rev. A 76, 012323 (2007).

[14] V. Händchen, T. Eberle, S. Steinlechner, A. Samblowski, T. Franz, R. F. Werner, and R. Schnabel, Nature Photonics 6, 596 (2012).

[15] G. Heinzel, K. A. Strain, J. Mizuno, K. D. Skeldon, B. Willke, W. Winkler, R. Schilling, A. Rüdiger, and K. Danzmann, Phys. Rev. Lett. 81, 5493 (1998).

[16] R. E. Slusher, L. W. Hollberg, B. Yurke, J. C. Mertz, and J. F. Valley, Phys. Rev. Lett. 55, 2409 (1985).

[17] R. M. Shelby, M. D. Levenson, S. H. Perlmutter, R. G. DeVoe, and D. F. Walls, Phys. Rev. Lett. 57, 691 (1986).

[18] L.-A. Wu, H. J. Kimble, J. L. Hall, and H. Wu, Phys. Rev. Lett. 57, 2520 (1986).

[19] K. McKenzie, N. Grosse, W. P. Bowen, S. E. Whitcomb, M. B. Gray, D. E. McClelland, and P. K. Lam, Phys. Rev. Lett. 93, 161105 (2004).

[20] H. Vahlbruch, S. Chelkowski, B. Hage, A. Franzen, K. Danzmann, and R. Schnabel, Phys. Rev. Lett. 97, 011101 (2006).

[21] H. Vahlbruch, S. Chelkowski, K. Danzmann, and R. Schnabel, New J. Phys. 9, 371 (2007).

[22] K. McKenzie, D. A. Shaddock, D. E. McClelland, B. C. Buchler, P. K. Lam, Phys. Rev. Lett. 88, 231102 (2002).

[23] H. Vahlbruch, S. Chelkowski, B. Hage, A. Franzen, K. Danzmann, R. Schnabel, Phys. Rev. Lett. 95, 211102 (2005).

[24] K. Goda, O. Miyakawa, E. E. Mikhailov, S. Saraf, R. Adhikari, K. McKenzie, R. Ward, S. Vass, A. J. Weinstein, and N. Mavalvala, Nature Phys. 4, 472 (2008).

[25] R. Schnabel, Nature Physics 4, 440, News\&Views (2008).

[26] A. Thüring, C. Gräf, H. Vahlbruch, M. Mehmet, K. Danzmann, R. Schnabel, Opt. Lett. 34, 824 (2009).

[27] Y. Takeno, M. Yukawa, H. Yonezawa, and A. Furusawa, Optics Express 15, 4321 (2007).

[28] H. Vahlbruch, M. Mehmet, S. Chelkowski, B. Hage, A. Franzen, N. Lastzka, S. Goßler, K. Danzmann, R. Schnabel, Phys. Rev. Let. 100, 033602 (2008).

[29] E. S. Polzik, Nature 453, 45, News\&Views (2008).

[30] T. Eberle, S. Steinlechner, J. Bauchrowitz, V. Händchen, H. Vahlbruch, M. Mehmet, H. MüllerEbhardt and R. Schnabel, Phys. Rev. Lett. 104, 251102 (2010).

[31] M. Mehmet, S. Ast, T. Eberle, S. Steinlechner, H. Vahlbruch, and R. Schnabel, Opt. Express 19, 25764 (2011).

[32] M. S. Stefszky, C. M. Mow-Lowry, S. S. Y. Chua, D. A. Shaddock, B. C. Buchler, H. Vahlbruch, A. Khalaidovski, R. Schnabel, P. K. Lam, and D. E. McClelland, Class. Quantum Grav. 29, 145015 (2012).

[33] H. Lück and the GEO 600 Team, Class. Quantum Grav. 14, 1471-1476 (1997). 
[34] B. Willke et al., Class. Quantum Grav. 19, 1377-1387 (2002).

[35] H. Grote et al., Class. Quantum Grav. 27, 084003 (2010).

[36] Henning Vahlbruch, PhD-thesis, Leibniz Universität Hannover, Germany (2008).

[37] Aleksandr Khalaidovski, PhD-thesis, Leibniz Universität Hannover, Germany (2011).

[38] A. Khalaidovski, H. Vahlbruch, N. Lastzka, C. Gräf, K. Danzmann, H. Grote and R. Schnabel, Class. Quantum Grav. 29, 075001 (2012).

[39] M. Prijatelj, J. Degallaix, H. Grote, J. Leong, C. Affeldt, S. Hild, H. Lück, J. Slutsky, H. Wittel, K. Strain, and K. Danzmann, Class. Quantum Grav. 29, 055009 (2012).

[40] H. Grote, K. Danzmann, K. L. Dooley, R. Schnabel, J. Slutsky, and H. Vahlbruch, Phys. Rev. Lett. 110, 181101 (2013).

[41] J. Abadie et al., Enhancing the astrophysical reach of the LIGO gravitational wave detector by using squeezed states of light, Nat. Photonics, accepted.

[42] M. Punturo et al., Class. Quantum Grav. 27, 084007 (2010).

[43] B. Sathyaprakash et al., Class. Quantum Grav. 29, 124013 (2012).

[44] K. Somiya (for the KAGRA Collaboration), Class. Quantum Grav. 29, 124007 (2012). 